\title{
Subjects no more: what happens when trial participants realize they hold the power?
}

\author{
() (1) $\Theta$ OPEN ACCESS \\ Patients will hold us all accountable in new and necessary ways
}

Paul Wicks vice president of innovation, Timothy Vaughan director of data science, James Heywood
cofounder and chairman

PatientsLikeMe, Cambridge, MA 02141, USA

The social contract of the randomized controlled trial is imbalanced: patients adhere to arduous protocols, are randomized to placebo, and are blinded to their health status. Although most participants ( $>90 \%)$ would like a lay summary of results, ${ }^{1}$ only a minority $(<10 \%)$ receive one, ${ }^{2}$ with the remainder left with the option of paying around $\$ 30$ (£18; €22) to read the results once the study is published in a peer reviewed journal. ${ }^{3}$ Such imbalances may have contributed to an emerging movement, enabled online by "patient powered research networks," in which participants have begun systematically to unblind themselves, pool their data, parse literature, conduct statistical analyses, and post their findings online.

In 2007, patients with amyotrophic lateral sclerosis (ALS) used Google to translate an Italian conference abstract suggesting that lithium carbonate might slow their illness. ${ }^{5}$ In a publication titled "Lithium delays progression of ALS" 16 patients treated with lithium (all of whom survived 15 months) were compared with 28 control patients (a third of whom did not survive the trial). ${ }^{6}$ Within six months of the abstract's publication 160 patients reported obtaining lithium off-label and tracked their progression using Google Spreadsheets and the validated ALS functional rating scale (ALSFRS-R). ${ }^{7}$ A patient in Brazil and a caregiver in the United States initiated this patient led study, raising the question of where ethical oversight lay. ${ }^{89}$

Our patient network, PatientsLikeMe, already allowed entry of ALSFRS-R scores but added tracking of lithium blood concentrations, data entry reminders, and monitoring by nurses to curate reported side effects. We presented data indicating that lithium was ineffective within nine months of the randomized controlled trial ${ }^{10}$ then published longer term follow-up data with more sophisticated analyses in an open access Nature Biotechnology paper, which included the entire de-identified dataset as supplementary material. ${ }^{11}$ Four randomized controlled trials subsequently replicated our negative findings. ${ }^{12}$

Shortly thereafter randomized controlled trials of two new drugs were under way: of NP001, manufactured by Neuraltus (in a phase II trial) and Biogen's dexpramipexole (phase III). Participants in these trials shared data while formally enrolled under protocols in which they were meant to be blinded and unaware of their ALSFRS-R score. They charted their own progress, seized on known side effects such as neutropenia in an attempt to unblind themselves, and used rudimentary statistics to analyze the efficacy of both drugs. Around a third of the total NP001 group and 10\% of US dexpramipexole patients recorded data online. A third experimental group was formed when some patients read the patents on NP001 and inferred that the industrial cleaner sodium chlorite might be the active ingredient. Some patients who could not enroll in the trial started ingesting industrial sodium chlorite orally or intravenously. ${ }^{13}$

Although we had data on fewer participants than for the lithium trial, we shared our analysis of all three groups through Figshare on the eve of the unblinding of the dexpramipexole trial. ${ }^{14}$ With important caveats, we estimated that dexpramipexole was below the cusp of providing a clinically significant benefit ${ }^{15}$ and NP001 just above it, but with confidence intervals that were too wide to draw a reliable conclusion (figure). Alarmingly, patients ingesting off-label sodium chlorite progressed worse than expected (figure). Biogen's dexpramipexole trial reported no effect, ${ }^{16}$ and funding is awaited for a phase III trial of NP001. When ALSUntangled used our data to warn against the potential dangers of sodium chlorite, its off-label use diminished. ${ }^{17}$ 


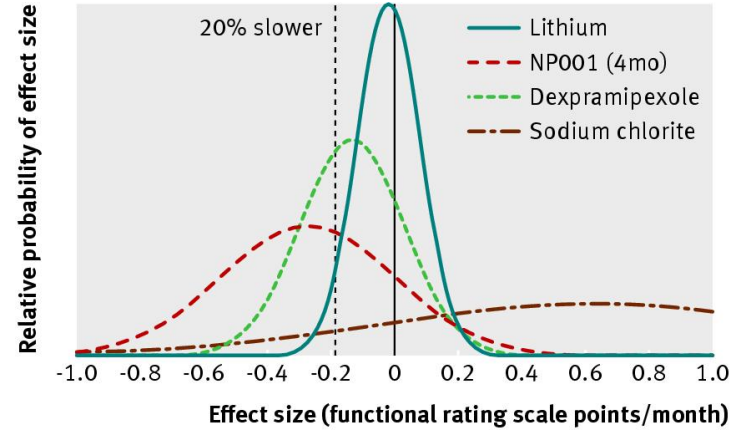

Estimates of effect size for selected ALS treatments. Each line represents the probability distribution of the effect size; a high, narrow peak indicates that the effect size is more precisely estimated (generally because of larger sample size). More effective treatments will be centered toward the left ${ }^{14}$

The concept of "scientific altruism" may be being trumped by "maximize your chance of survival." For better or worse, digital tools enable greater self knowledge and rapid dissemination. The consequence is that scientific design, informed consent, and ethical oversight can be short circuited by patient led "disobedience." Some drug companies will choose to share their clinical trial data (as AllTrials suggests), but even if they don't the data can become available if participants choose to share their data themselves, something that will only be enhanced by patient access to electronic medical records. Today members of PatientsLikeMe report tracking their outcomes in over 400 randomized trials. Patients increasingly realize that they are both statistically and literally the "power" in trials and we need to build systems that redress the imbalance. If we collectively do nothing, a phase III study might be rendered scientifically null by a critical mass of participants making intentional protocol violations on PatientsLikeMe, Facebook, or Twitter.

This would be a tragic outcome. To prevent that, we propose forging a new social contract that maximizes both scientific discovery and patient autonomy, setting the stage for better trials with more engaged participants. Together we can develop rigorous new methods to include patients in selecting therapies, protocol design, recruitment, feedback, lay summaries, publications, and assessment of value. We are encouraged by the development of an online "open research exchange" that allows researchers rapid access to patients for concept elicitation and psychometric validation during the development of patient reported outcome measures, ${ }^{18}$ which are now required by the FDA. ${ }^{19}$ We believe that patients may surprise many of us with their ability to identify obstacles to trial enrolment, prioritize the outcomes they truly value, and help us learn what works in the real world, not just in trials. With the new tools at their disposal patients will hold us all accountable in new and necessary ways. Patients themselves have already laid much of the groundwork; let's ask them to continue building on these new systems together as equals.

Competing interests: We have read and understood the BMJ policy on declaration of interests and declare PW, TV, and JH are employees of PatientsLikeMe and own stock or stock options in the company. The PatientsLikeMe research and development team has received research support from pharmaceutical companies and private foundations.

Provenance and peer review: Not commissioned; not externally peer reviewed.

Partridge AH, Winer EP. Sharing study results with trial participants: time for action. $J$ Clin Oncol 2009;27:838-9.

2 Cox K, Moghaddam N, Bird L, Elkan R. Feedback of trial results to participants: a survey of clinicians' and patients' attitudes and experiences. Eur J Oncol Nurs 2011;15:124-9.

3 Science Direct. Pay per view. http://info.sciencedirect.com/sciencedirect/buying/individual article purchase options/ppv.

4 Workman TA. Engaging patients in information sharing and data collection: the role of patient-powered registries and research networks. US Agency for Healthcare Research and Quality, 2013.

5 Frost JH, Massagli MP, Wicks P, Heywood J. How the social web supports patient experimentation with a new therapy: The demand for patient-controlled and patient-centered informatics. AMIA Annu Symp Proc 2008:217-21.

6 Fornai F, Longone P, Cafaro L, Kastsiuchenka O, Ferrucci M, Manca ML, et al. Lithium delays progression of amyotrophic lateral sclerosis. Proc Natl Acad Sci USA 2008;105:2052-7.

7 Cedarbaum JM, Stambler N, Malta E, Fuller C, Hilt D, Thurmond B, et al. The ALSFRS-R a revised ALS functional rating scale that incorporates assessments of respiratory function. J Neurol Sci 1999:169:13-21.

8 O'Connor D. The apomediated world: regulating research when social media has changed research. J Law Med Ethics 2013;41:470-83.

Vayena E, Tasioulas J. Adapting standards: ethical oversight of participant-led health research. PLOS Med 2013;10:e1001402.

10 Wicks P, Massagli M, Frost J, Macedo H, Felzer K, Heywood J. A patient-led trial of lithium in ALS using the internet. Amyotroph Lateral Scler 2008:9:S59.

11 Wicks P, Vaughan TE, Massagli MP, Heywood J. Accelerated clinical discovery using self-reported patient data collected online and a patient-matching algorithm. Nat Biotechnol 2011;29:411-4.

12 Armon C. Is the lithium-for-ALS genie back in the bottle? Not quite. Neurology 2010;75:586-7.

13 Dokser Marcus A. Frustrated ALS patients concoct their own drug. Wall St Journal 2012 Apr 15. http://online.wsj.com/news/articles/ SB10001424052702304818404577345953943484054.

14 Heywood J. Waiting for $\mathrm{P}<0.05$. Figshare 2012. http://dx.doi.org/10.6084/m9.figshare. 96802

15 Castrillo-Viguera C, Grasso DL, Simpson E, Shefner J, Cudkowicz ME. Clinical significance in the change of decline in ALSFRS-R. Amyotroph Lateral Scler 2010;11:178-80.

16 Cudkowicz ME, van den Berg LH, Shefner JM, Mitsoumuto H, Mora JS, Ludolph A, et al Dexpramipexole versus placebo for patients with amyotrophic lateral sclerosis (EMPOWER): a randomised, double-blind, phase 3 trial. Lancet Neurol 2013;12:1059-67.

17 ALSUntangled Group. ALSUntangled No 19: Sodium chlorite. Amyotroph Lateral Scler Frontotemporal Degener 2013;14:236-8.

18 Wicks $\mathrm{P}$, Heywood B, Heywood J. Online platform to accelerate patient involvement in open instrument development Qual Life Res 2013.22:1-137.

19 Gnanasakthy A, Mordin M, Clark M, DeMuhro C, Fehnel S, Copley-Merriman C. A review of patient-reported outcome labels in the United States: 2006 to 2010. Value Health 2012;15:437-42.

\section{Cite this as: BMJ 2014;348:g368}

This is an Open Access article distributed in accordance with the Creative Commons Attribution Non Commercial (CC BY-NC 3.0) license, which permits others to distribute, remix, adapt, build upon this work non-commercially, and license their derivative works on different terms, provided the original work is properly cited and the use is non-commercial. See: http://creativecommons.org/licenses/by-nc/3.0/. 